\title{
Zitterbewegung in External Magnetic Field: Classic versus Quantum Approach
}

\author{
Mehran Zahiri-Abyaneh* and Mehrdad Farhoudi ${ }^{\dagger}$ \\ Department of Physics, Shahid Beheshti University, G. C., Evin, Tehran 19839, Iran
}

February 25, 2011

\begin{abstract}
We investigate variations of the Zitterbewegung frequency of electron due to an external static and uniform magnetic field employing the expectation value quantum approach, and compare our results with the classical model of spinning particles. We demonstrate that these two so far compatible approaches are not in agreement in the presence of an external uniform static magnetic field, in which the classical approach breaks the usual symmetry of free particles and antiparticles states, i.e. it leads to $\mathrm{CP}$ violation. Hence, regarding the Zitterbewegung frequency of electron, the classical approach in the presence of an external magnetic field is unlikely to correctly describe the spin of electron, while the quantum approach does, as expected. We also show that the results obtained via the expectation value are in close agreement with the quantum approach of the Heisenberg picture derived in the literature. However, the method we use is capable of being compared with the classical approach regarding the spin aspects. The classical interpretation of spin produced by the altered Zitterbewegung frequency, in the presence of an external magnetic field, are discussed.
\end{abstract}

PACS number: 03.65.Pm; 03.65.-w; 03.65.Sq; 14.60.Cd

Keywords: Zitterbewegung; Relativistic Quantum Mechanics; Spinning Particles

\section{Introduction}

As velocity and momentum in the Dirac theory [1] are related in a different manner than in nonrelativistic mechanics, it leads [2]-[8] to the fact that the average speed of spin- $1 / 2$ particles must be less than $c$, while the instantaneous speed is always $\pm c$ in this theory. Indeed, by using an arbitrary state of a spin-1/2 free particle in which it is localized in space, one can show [9] that the expectation value of the position, even in the absence of external field, includes a time-dependent part which originates from an interference between the positive and negative energy states. Hence, the motion of free particle has a peculiar oscillatory component with a very high frequency of $2 m c^{2} / \hbar \simeq 1.6 \times 10^{21} \mathrm{~s}^{-1}$ for electrons with the rest mass $\mathrm{m}$ - which secures $\pm c$ as the eigenvalues of instantaneous speed. That is, a free particle obeys a rapid oscillatory motion with the speed $c$ around the center of mass while moving like a relativistic particle with velocity $\mathbf{p} / m$. This angular frequency is referred to $\omega_{\text {zbw }} \equiv 2 m c^{2} / \hbar$ after Schrödinger who called this motion Zitterbewegung (Z Zbw) $[10,11]$ - trembling or quivering motion - which he theoretically contrived it after Dirac had employed his equation for free relativistic electron in vacuum. He interpreted this as a fluctuation in the position of electron. The amplitude of the $\mathrm{Zbw}$ has also been indicated to be of the order of the Compton wavelength, $\lambda_{c}=\hbar /(m c) \simeq 3.86 \times 10^{-13} \mathrm{~m}$ for electrons. Now, the well-known phenomenon of the Zbw, caused

*mehran.z.abyaneh@gmail.com

${ }^{\dagger}$ m-farhoudi@sbu.ac.ir 
by the interference between the positive and negative energy components of a wave packet, has been described in many textbooks as well [2]-[8].

It is also well-known [2]-[8] that as the position operator in the Dirac theory is not a one-particle observable, the path of an electron can only be indicated up to the order of its Compton wavelength. Indeed, the best localized wave packet exclusively consisting of plane wave components with positive or negative energy has characteristic linear dimension of about $\lambda_{c}$ [12]. However, the $\mathrm{Zbw}$ for free electrons is not an observable motion and has never been directly detected [13] (nevertheless, see discussion in Sec. 3). Actually, with certain initial conditions, one can show [9] that all components of $\langle\boldsymbol{r}\rangle$ vanish upon integration, which indicates that the total contribution of all waves vanishes (see Sec. 2). Hence, in these conditions, the center of the wave packet as a whole remains at rest, as experimental evidence rules out the possibility that electron is an extended body! Though, the consequence of the $\mathrm{Zbw}$ is that it is impossible to localize electron better than to a certain finite volume.

The above argument leaves open the possibilities that electron may be regarded as a point charge which generates spin and magnetic moment by an intrinsic local motion [15]. Actually, in the literature, people have tried to analyze some picture about the detailed motion of the Zbw and to interpret the structural feature of electron. The motivation has mainly come from the close connection between the $\mathrm{Zbw}$ and the intrinsic magnetic moment of electron. That is, using the current density expression of a free electron, one can infer $[16,17]$ that the classical expression for the magnetic moment has its root in the Zbw (see also Sec. 2). Hence, a temptation that the magnetic moment and the spin of electron are generated by some sort of the local circular motion of electron, i.e. mass and charge, $[9,15,18,19,20]$.

Indeed, many classical treatments of a free Dirac electron have been formed based on this picture for the Zbw, see Refs. [21]-[25] and references therein. A significant achievement toward understanding the Zbw has been made [26]-[28] when it was shown that in the absence of external fields the Dirac Hamiltonian can be transformed into a form in which the positive and negative electron energies are decoupled. In this new representation, electron is not a point-like particle and has the size of about $\lambda_{c}$. The interpretation of these two pictures has been considered in Refs. [9, 11, 12], [29]-[32], and the compatibility of the Zbw interpretation with the details of the Dirac theory has also been demonstrated [19, 20,33].

Huang [9] obtained this picture from the Dirac equation and claimed that it is not an unreasonable one. Actually, using an explicit form for the wave packet with specified initial condition to construct the wave packet mainly from positive or negative waves, he showed that the contribution of each plane wave to the motion of the wave packet is an orbit whose projection on the plane perpendicular to the direction of the spin is a circle of radius $\lambda_{c} / 2$. Then, using the method of the Gordon decomposition $[16,17]$, he also justified that the intrinsic spin of electron may be considered as an "orbital angular momentum" due to the Zbw. That is, the current produced by the Zbw actually causes the intrinsic magnetic moment of the particle, and hence, the total magnetic moment of electron is represented by both the orbital and the intrinsic angular momenta with the correct gyromagnetic $g$ factor.

A classical analogous model for spinning electron, which has been claimed to be a natural classical limit of the Dirac theory [25], has been presented [33] by Barut and Zanghi (BZ) in a particular five dimensional space-time (which we refer to as BZ approach). They introduced a classical Lagrangian (see relation (45)) in a manifestly covariant form using a pair of spinorial variables (representing internal degrees of freedom) besides the usual pair of dynamical variables $\left(x^{\mu}, p^{\mu}\right)$, as functions of the proper time parameter measured in the center of mass frame (see Refs. [25, 34] and references therein). The general solution of the motion for free electrons includes an oscillatory part as well, which denotes the presence of the Zbw for the spinning particles (see Sec. 3). That is, the Zbw occurs because the motion of particle does not coincide with the motion of the center of mass [35], and a

\footnotetext{
${ }^{1}$ Indeed, scattering experiments have revealed that the size of electron is less than $10^{-18} \mathrm{~m}[14]$.

${ }^{2}$ The same issue, but in the presence of an external magnetic field, is studied at the end of Sec. 2.
} 
spinning particle appears as extended object [34]. It has also been found that free polarized particles have circular motions in the plane perpendicular to the direction of the spin, with the Zbw frequency and half the Compton wavelength radius in the center of mass frame.

After quantization, the BZ approach has also been indicated to describe the Dirac electron [36]. In fact, this has been originated from the classical Hamiltonian corresponding to a classical Lagrangian devised by BZ [33] (here shown with relation (45)). Then, it has been converted to a quantum version with reinterpretation of the spinorial variables as Dirac field spinors [37, 38]. Also, by recasting the BZ approach into the language of Clifford formalism, quantization of the theory has been accomplished [38].

The significant correspondence between the Dirac theory and BZ approach is intriguing and invites one to discover up to where this compatibility lasts, and actually whether there is not any conflict between them. To pursue such a query, we purpose to study the particle's Zbw in the presence of an external uniform static magnetic field. Our stimulated hope in exploring this procedure is that theoretical understanding of the Zbw may shed light on the nature of spin of elementary particles.

In the standard textbooks [2]-[8], the Zbw has only been examined in details for free spinning particles without considering any interactions. Though, it has been indicated that electron in the hydrogen atom, i.e. in the presence of the Coulomb potential, also displays the Zbw which gives rise to the so called Darwin term [2]-[8]. Interestingly enough, the phenomenon of Zbw for electrons has been shown [13], [39]-[51] to occur in non-relativistic case and in solids in the absence of external fields. Even, an analogous quantum-relativistic effect in a single trapped ion has been studied [52]. In these conditions, it has much lower angular frequency and much larger amplitude, and it may lead easier to experimental detection (also see Sec. 3). Also, in Refs. [25, 33, 53] and intuitively in Ref. $[54,55]$, the phenomenon of $\mathrm{Zbw}$ for electrons in the presence of an external magnetic field has been established. Hence, the $\mathrm{Zbw}$ is not a special characteristic of plane wave solution, and does occur whenever positive and negative energy states are mixed [19, 53].

Actually, by interpreting the interference between positive and negative energy states, the Zbw may be considered to arise in electron-positron pair creation and annihilation [56] - which manifests the $\mathrm{Zbw}$ as a purely relativistic phenomenon. On the other hand, the $\mathrm{Zbw}$ is considered to be the origin of the spin only in the non-relativistic domain. By reformulating the Dirac theory, it has been claimed $[19,20]^{4}$ that the Zbw is a phenomenon which manifests itself in every application of quantum mechanics, i.e. either in the non-relativistic domain and/or in the presence of an arbitrary electromagnetic interaction. Indeed, it has been claimed $[15,19]$ that the most features of quantum mechanics are manifestations of the Zbw, e.g. the complex phase factor in the electron wave function as physical representation of rotation can be associated directly with the Zbw. However, we should mention the assertion [32] that quantum field theory prohibits the occurrence of the Zbw for an electron, and in principle, there is no difficulty to localize an electron narrower than the Compton wavelength if it is involved with a positron.

Regarding the question of an electron in vacuum in the presence of an external magnetic field, the references [25] and [53] are of much interest to us. This is in fact the topic that we purpose to deal with by making recourse to the Huang quantum approach [9] where the next section is devoted to. There, we contrast the results with those two investigations, particularly with the more accurate solutions that we will indicate by using Ref. [25] in where the BZ classical approach has been employed to study the issue. Actually, in Sec. 2, the expectation value approach has been utilized to find changes of $\omega_{\mathrm{zbw}}$ in an external uniform static magnetic field. In Sec. 3, we review the classical approach of Ref. [25] and infer some improvements. It will be shown that the results derived via the expectation value quantum approach are different from the corresponding ones obtained via the classical approach from Ref. [25]. Finally, a brief discussion and conclusion is given in the last section.

\footnotetext{
${ }^{3}$ In which they have also claimed that this algebra allows various independent classical approaches of the spin to be unified.

${ }^{4}$ Also, see Ref. [38].
} 


\section{Frequency Shift via Expectation Value Approach}

Huang [9] used solutions to the Dirac equation and the expectation value of velocity to study the issue of free electron's Zbw. In this work, we evaluate the electron's Zbw in the presence of an external uniform static magnetic field utilizing his method. Thus, we first very briefly review some results for the free particle and then, we will extend the method to our case.

To examine the average position of an electron, which is localized in space, a general Dirac wave packet, expanded in terms of momentum eigenfunctions, as

$$
\Psi(\boldsymbol{r}, t)=h^{-3 / 2} \int\left[C_{+}(\mathbf{p}) \exp (-i \omega t)+C_{-}(\mathbf{p}) \exp (i \omega t)\right] \exp \left(i \frac{\mathbf{p} \cdot \boldsymbol{r}}{\hbar}\right) d^{3} p
$$

can be used. This wave packet includes both negative and positive energies, and $C_{+}(\mathbf{p})\left(C_{-}(\mathbf{p})\right)$ is a linear combination of the spin-up and spin-down amplitudes of the free particle Dirac waves with momentum $\mathbf{p}$ and positive (negative) energy. Using this wave packet and calculating $<\dot{\boldsymbol{r}}>$, and consequently $\langle\boldsymbol{r}>$ with a suitable initial condition having a well-defined initial spin direction, it has been shown [9] that each plane wave in the above Fourier decomposition contributes a circular motion in the plane perpendicular to the direction of the electron spin with radius $\lambda_{c} / 2$ and frequency $\omega_{\mathrm{zbw}}$. This leads to the internal spin and hence, the magnetic moment of the Dirac particle.

Now, the Dirac equation for an electron in an external electromagnetic field is

$$
i \hbar \frac{\partial \Psi}{\partial t}=\left[c \alpha_{i}\left(p^{i}-\frac{e}{c} A^{i}\right)+\beta m c^{2}+e A_{0}\right] \Psi
$$

where the matrices $\boldsymbol{\alpha}$ and $\beta$ are defined as $^{5}$

$$
\alpha_{i}=\left(\begin{array}{cc}
0 & \sigma_{i} \\
\sigma_{i} & 0
\end{array}\right) \quad \text { and } \quad \beta=\left(\begin{array}{cc}
I & 0 \\
0 & -I
\end{array}\right),
$$

with $\sigma_{i}$ and $I$ as the $2 \times 2$ Pauli matrices and the unit matrix, respectively. We proceed with special case of an external uniform static magnetic field without a scalar Coulomb field (i.e. $A_{0}=0$ ). Then, using the standard abbreviation $\pi_{i} \equiv p_{i}-e A_{i} / c$ in the Gaussian units - i.e. defining the gaugeinvariant momentum operator correspondence $\pi_{\mu} \rightarrow-i \hbar D_{\mu}$ in terms of the covariant derivative ${ }^{6}-$ the Dirac equation (2) reads $i \hbar \partial \Psi / \partial t=\left(c \alpha_{i} \pi^{i}+\beta m c^{2}\right) \Psi$. Making a general wave solution ansatz ${ }^{7}$

$$
\Psi(\boldsymbol{r}, t)=\left(\begin{array}{c}
\phi(\boldsymbol{r}) \\
\chi(\boldsymbol{r})
\end{array}\right) \exp -i\left(\frac{E_{\mathrm{B}} t}{\hbar}\right),
$$

with $E_{\mathrm{B}}$ as the particle energy in the presence of an external magnetic field, hence the Dirac equation yields

$$
\left(E_{\mathrm{B}}+m c^{2}\right) \chi=c \sigma_{i} \pi^{i} \phi \quad \text { and } \quad\left(E_{\mathrm{B}}-m c^{2}\right) \phi=c \sigma_{i} \pi^{i} \chi .
$$

A uniform magnetic field is derivable from a vector potential $\mathbf{A}=(\mathbf{B} \times \boldsymbol{r}) / 2$, which for $\mathbf{B}$ along the $z$-direction, becomes $\mathbf{A}=-(y B \hat{x}-x B \hat{y}) / 2$. Such a field, without loss of generality, can also be achieved by choosing a vector potential as $A_{x}=0=A_{z}$ and $A_{y}=x B$. This way, one can make ansatz [6]

$$
\phi(\boldsymbol{r})=h(x) \exp \left[i\left(p_{y} y+p_{z} z\right) / \hbar\right]
$$

to eliminate $\chi$ in equations (5) and then finds that $h(\xi) \propto H_{n}(\xi) \exp \left(-\xi^{2} / 2\right)$, where $H_{n}$ represents the Hermit polynomials. Noting that positive energy corresponds to electron with negative charge

\footnotetext{
${ }^{5}$ In terms of the Dirac (gamma) matrices, $\gamma_{a}$, they are $\beta \equiv \gamma^{0}$ and $\alpha_{i} \equiv \gamma^{0} \gamma_{i}$ for $i=1,2,3$.

${ }^{6}$ In a general case, the rate of change of this generalized operator, via the Heisenberg picture, gives the Lorentz force, i.e. $\dot{\boldsymbol{\pi}}=i[H, \boldsymbol{\pi}] / \hbar-(e / c) \partial \mathbf{A} / \partial t=e(\mathbf{E}+\boldsymbol{\alpha} \times \mathbf{B})$.

${ }^{7}$ We should emphasis that due to the presence of an external magnetic field the wave packet $\Psi$ is not an eigenfunction of neither the operator $\pi_{i}$ nor the operator $p_{i}$.
} 
$(-|e|)$ while negative energy describes positron with positive charge $(|e|)$, and taking the sign of $B$ such that $-|e| B>0$, one has $\xi=\sqrt{-|e| B / c}\left(x \pm c p_{y} /|e| B\right)$ with the plus (minus) sign for positive (negative) energy.

Thus, the energy spectrum of a particle with a magnetic moment $\boldsymbol{\mu}$ in a strong external magnetic field along the $z$-direction, employing the cylindrical coordinates and neglecting back-reaction of the motion of charge on the field, takes the form $[6,54]$

$$
E_{\mathrm{ln}}^{2}=m^{2} c^{4}+p_{z}^{2} c^{2}+c e B\left(n-l+1-2 s_{z}\right),
$$

where $p_{z}=\hbar k, s_{z}= \pm \hbar / 2, n$ is a non-negative integer and $L_{z}=\hbar l$, while $l$ is restricted to values $l=-n,-n+2, \cdots, n-2, n$.

The term $n-l+1$ represents contribution from the orbital part of the magnetic moment, whereas we purpose to deal with effects of an external magnetic field only on the internal spin. Thus, from the spectrum (7), one can infer [55]

$$
E_{\mathrm{B}}^{2}=m^{2} c^{4}+p_{z}^{2} c^{2}-2 c e B s_{z} .
$$

Defining $\sigma_{ \pm}= \pm 1$ as the eigenvalues of the operator $\sigma_{z}$ with positive (negative) sign representing the up (down) spin state, and reminding that positive (negative) energy relates to electron (positron), relation (8), in the weak field approximation and non-relativistic limit (justified below Eq. (21)), reads

$$
\frac{E_{\mathrm{B} \pm}}{\hbar} \simeq \pm\left(\frac{m c^{2}}{\hbar} \pm \frac{|e| B \sigma_{ \pm}}{2 m c}\right) \equiv \pm\left(\omega \pm \Omega \sigma_{ \pm}\right),
$$

where $\omega \equiv \omega_{\mathrm{zbw}} / 2, \Omega \equiv|e| B / 2 m c=\omega_{c} / 2, \omega_{c}$ is the usual classical cyclotron frequency. The weak field approximation means $\Omega \ll \omega$ or $\omega_{c} \ll \omega_{\mathrm{zbw}}$, which is a reasonable approximation even with the highest magnetic field achievable in the current laboratories. One should also note that, having the external magnetic field along the $z$-direction with the proposed vector potential, the only altered generalized momentum is $\pi_{y}$, while $\pi_{z}=p_{z}$ and $\pi_{x}=p_{x}$. In addition, in order to justify use of Eq. (7) and also simplify later on calculations, we ignore the non-commutativity of $\pi_{x}$ and $\pi_{y}$ (justified below Eq. (20)).

As we are interested to find effects of an external magnetic field on the $\omega_{\mathrm{zbw}}$, following Huang's approach, it is more appropriate and instructive to solve these equations by the initial spinoral eigenstates. That is, using $\phi^{\uparrow}=\left(h_{+}^{\uparrow}, 0\right)$ and $\phi^{\downarrow}=\left(0, h_{+}^{\downarrow}\right)$ as the initial positive energy eigenstates for the spin-up and spin-down, and $\chi^{\uparrow}=\left(h_{-}^{\uparrow}, 0\right)$ and $\chi^{\downarrow}=\left(0, h_{-}^{\downarrow}\right)$ as the initial negative energy eigenstates for the spin-up and spin-down, respectively. Then, by relation (9) and ansatz (6), a general solution of Eqs. (5) becomes

$$
\begin{gathered}
\Psi(\boldsymbol{r}, t)=h^{-3 / 2} \int\left\{\mathcal{A}\left(\begin{array}{c}
\phi^{\uparrow} \\
k_{+}^{\uparrow} \boldsymbol{\sigma} \cdot \boldsymbol{\pi} \phi^{\uparrow}
\end{array}\right) \exp [-i(\omega+\Omega) t]+\mathcal{B}\left(\begin{array}{c}
\phi^{\downarrow} \\
k_{+}^{\downarrow} \boldsymbol{\sigma} \cdot \boldsymbol{\pi} \phi^{\downarrow}
\end{array}\right) \exp [-i(\omega-\Omega) t]\right. \\
\left.+\mathcal{C}\left(\begin{array}{c}
k_{-}^{\uparrow} \boldsymbol{\sigma} \cdot \boldsymbol{\pi} \chi^{\uparrow} \\
\chi^{\uparrow}
\end{array}\right) \exp [i(\omega-\Omega) t]+\mathcal{D}\left(\begin{array}{c}
k_{-}^{\downarrow} \boldsymbol{\sigma} \cdot \boldsymbol{\pi} \chi^{\downarrow} \\
\chi^{\downarrow}
\end{array}\right) \exp [i(\omega+\Omega) t]\right\} \exp \left[i\left(p_{y} y+p_{z} z\right) / \hbar\right] d^{3} \pi,
\end{gathered}
$$

where $\mathcal{A}, \mathcal{B}, \mathcal{C}$ and $\mathcal{D}$ are complex functions of $\boldsymbol{\pi}$ and $k$ 's. The notation $k_{ \pm}$for positive and negative energies in the absence of external fields are $k_{ \pm}= \pm c /\left(E+m c^{2}\right)$ with $E=\left(p^{2} c^{2}+m^{2} c^{4}\right)^{1 / 2}[9]$, while in our situation, with the aid of relation (9), it reads

$$
k_{-}^{\uparrow}=-k_{+}^{\downarrow}=\frac{-c}{2 m c^{2}-\hbar \Omega} \equiv-K_{1} \quad \text { and } \quad k_{-}^{\downarrow}=-k_{+}^{\uparrow}=\frac{-c}{2 m c^{2}+\hbar \Omega} \equiv-K_{2} .
$$

More explicitly, comparing solution (10) with the one in the absence of magnetic field - namely the plane wave packet (1) - reveals that one has

$$
\begin{cases}\exp (i \omega t) \rightarrow \exp [i(\omega-\Omega) t] & \text { for negative energy } \\ \exp (-i \omega t) \rightarrow \exp [-i(\omega+\Omega) t] & \text { for positive energy }\end{cases}
$$


for the spin-up states, and

$$
\begin{cases}\exp (i \omega t) \rightarrow \exp [i(\omega+\Omega) t] & \text { for negative energy } \\ \exp (-i \omega t) \rightarrow \exp [-i(\omega-\Omega) t] & \text { for positive energy }\end{cases}
$$

for the spin-down states. Let us write the wave packet (10) in the form

$$
\begin{aligned}
\Psi(\boldsymbol{r}, t) & =h^{-3 / 2} \int\left\{C_{+}^{\uparrow}(\boldsymbol{\pi}) h_{+}^{\uparrow} \exp [-i(\omega+\Omega) t]+C_{+}^{\downarrow}(\boldsymbol{\pi}) h_{+}^{\downarrow} \exp [-i(\omega-\Omega) t]\right. \\
& \left.+C_{-}^{\uparrow}(\boldsymbol{\pi}) h_{-}^{\uparrow} \exp [i(\omega-\Omega) t]+C_{-}^{\downarrow}(\boldsymbol{\pi}) h_{-}^{\downarrow} \exp [i(\omega+\Omega) t]\right\} \exp \left[i\left(p_{y} y+p_{z} z\right) / \hbar\right] d^{3} \pi,
\end{aligned}
$$

where

$$
C_{+}^{\uparrow}(\boldsymbol{\pi})=\mathcal{A}\left(\begin{array}{c}
1 \\
0 \\
K_{2} \pi_{z} \\
K_{2} \pi_{+}
\end{array}\right), \quad C_{+}^{\downarrow}(\boldsymbol{\pi})=\mathcal{B}\left(\begin{array}{c}
0 \\
1 \\
K_{1} \pi_{-} \\
-K_{1} \pi_{z}
\end{array}\right), \quad C_{-}^{\uparrow}(\boldsymbol{\pi})=\mathcal{C}\left(\begin{array}{c}
-K_{1} \pi_{z} \\
-K_{1} \pi_{+} \\
1 \\
0
\end{array}\right), \quad C_{-}^{\downarrow}(\boldsymbol{\pi})=\mathcal{D}\left(\begin{array}{c}
-K_{2} \pi_{-} \\
K_{2} \pi_{z} \\
0 \\
1
\end{array}\right),
$$

where $\pi_{ \pm} \equiv \pi_{x} \pm i \pi_{y}$. Implicitly, the normalization condition of the wave packet (14) is provided if one requires that

$$
\int\left[\left|C_{+}^{\uparrow *}(\boldsymbol{\pi})\right|^{2}+\left|C_{-}^{\uparrow *}(\boldsymbol{\pi})\right|^{2}+\left|C_{+}^{\downarrow *}(\boldsymbol{\pi})\right|^{2}+\left|C_{-}^{\downarrow *}(\boldsymbol{\pi})\right|^{2}\right] d^{3} \pi=1
$$

In order to write the exact forms of $C_{ \pm}^{\uparrow \downarrow}(\boldsymbol{\pi})$, one needs to have an explicit expression for $\Psi$, which leads to evaluation of $\langle\dot{\boldsymbol{r}}\rangle=\mathrm{c}\langle\boldsymbol{\alpha}\rangle$. For this purpose, we take the initial state of the wave packet as a localized spin-up electron in the $z$-direction while its center is at rest in the origin, namely

$$
\Psi(\boldsymbol{r}, 0)=\left(\begin{array}{c}
1 \\
0 \\
0 \\
0
\end{array}\right) f\left(\frac{r}{r_{o}}\right)
$$

where $f\left(r / r_{o}\right)=\left[2 /\left(\bar{\pi} r_{o}^{2}\right)\right]^{3 / 4} \exp \left[-\left(r / r_{o}\right)^{2}\right]$ is a normalized Gaussian function with $r \equiv|r|$ and $r_{o}$ as a constant that indicates approximate spatial extension of the wave packet. The notation $\bar{\pi}$ has been introduced to indicate the usual pi number in order not to be confused with the generalized momentum $\boldsymbol{\pi}$. The Fourier transformation of $f\left(r / r_{o}\right)$ is

$$
f\left(\frac{\pi}{\pi_{o}}\right)=\left(\frac{2}{\bar{\pi} \pi_{o}^{2}}\right)^{3 / 4} \exp \left[-\left(\frac{\pi}{\pi_{o}}\right)^{2}\right]
$$

which, taking $\boldsymbol{\pi}$ in the spherical coordinates with $\boldsymbol{\pi}=\boldsymbol{\pi}(\pi, \theta, \varphi)$, satisfies the normalization condition $\int f^{2}\left(\pi / \pi_{o}\right) d^{3} \pi=1$, and where the constant $\pi_{o}=2 \hbar / r_{o}$ gives the width of wave packet in the momentum space [9].

As we have applied the non-relativistic approximation, to simplify the model further, we drop terms of the order $\epsilon^{2} \equiv(\pi / 2 m c)^{2}$ or higher. Thus, in the weak field approximation, $\Omega \ll \omega$, one has $(\pi / 2 m c)\left(\hbar \Omega / 2 m c^{2}\right) \approx \mathcal{O}\left(\epsilon^{2}\right)$ or higher, and then from (11) one can write

$$
\left(\begin{array}{l}
K_{1} \\
K_{2}
\end{array}\right) \pi \simeq \frac{\pi}{2 m c}\left(\begin{array}{c}
1+\frac{\hbar \Omega}{2 m c^{2}} \\
1-\frac{\hbar \Omega}{2 m c^{2}}
\end{array}\right) \simeq \frac{\pi}{2 m c}\left(\begin{array}{l}
1 \\
1
\end{array}\right) \equiv K \pi\left(\begin{array}{l}
1 \\
1
\end{array}\right) .
$$


In addition, during the calculation one encounters non-commutative terms due to the existence of the magnetic field. For our choice of vector potential, when $\pi_{i}$ 's are operators (i.e. not integration variables), one gets

$$
\pi_{+} \pi_{-}-\pi_{-} \pi_{+}=2 i\left(\pi_{y} \pi_{x}-\pi_{x} \pi_{y}\right)=\frac{2 e B \hbar}{c} .
$$

Therefore, up to the order of approximation we are dealing with, the non-commutativity of $\pi_{x}$ and $\pi_{y}$ can be ignored, as assumed before, and we use $\pi_{+} \pi_{-} \simeq \pi_{-} \pi_{+}$in the following. Also, regarding the fact that $h_{ \pm}^{\uparrow \downarrow}(\xi) \propto H_{n}(\xi) \exp \left(-\xi^{2} / 2\right)$, one gets $\left(\pi_{x} h_{ \pm}^{\uparrow \downarrow}-h_{ \pm}^{\uparrow \downarrow} \pi_{x}\right)$ to be of the order $\hbar \sqrt{e B / c}$, where it can also be ignored in the proposed approximation. Hence, using rough coefficients (A.1) for the initial state (17), the wave packet (14) reads

$$
\begin{aligned}
\Psi(\boldsymbol{r}, t) & \simeq h^{-3 / 2} \int\left\{\left(\begin{array}{c}
1 \\
0 \\
K \pi_{z} \\
K \pi_{+}
\end{array}\right) \exp [-i(\omega+\Omega) t]+\left(\begin{array}{c}
0 \\
0 \\
-K \pi_{z} \\
0
\end{array}\right) \exp [i(\omega-\Omega) t]\right. \\
& \left.+\left(\begin{array}{c}
0 \\
0 \\
0 \\
-K \pi_{+}
\end{array}\right) \exp [i(\omega+\Omega) t]\right\} f\left(\pi / \pi_{o}\right) \exp \left[i\left(p_{y} y+p_{z} z\right) / \hbar\right] d^{3} \pi .
\end{aligned}
$$

Note that, each of the above rough solutions satisfy the Dirac equation (2) when the aforementioned approximations are taken into account. Actually, by the exact coefficients (A.1), they do fulfill the Dirac equation exactly, and our approximations do not alter the results for altered Zbw, rather they simplify calculations.

Since the wave function must not spread rapidly in time, one can justify the non-relativistic approximation applied in (19) if $r_{o}$ is taken to be very large compared to the Compton wavelength [9] or equally $\pi_{o} / m c \ll 1$. Therefore, all momenta which are greater than $\pi_{o}$ are diminished in the integrand (21), and hence, the main role is played by momenta that satisfy $\pi / m c \ll 1$. This justifies the non-relativistic approximation.

Now, making use of the expectation value formula for velocity vector,

$$
<\dot{\boldsymbol{r}}>=\int \Psi^{*}(\boldsymbol{r}, t)(c \boldsymbol{\alpha}) \Psi(\boldsymbol{r}, t) d^{3} r
$$

with the wave packet (21), it is straightforward to show that for an initially localized spin-up electron

$$
<\dot{\boldsymbol{r}}>=\mathbf{V}+2 c \int\left\{l_{1}(\boldsymbol{\pi}) \cos \left[\left(\omega_{\mathrm{zbw}}+\omega_{c}\right) t+\varphi(\boldsymbol{\pi})\right]+l_{2}(\boldsymbol{\pi}) \cos \omega_{\mathrm{zbw}} t\right\} d^{3} \pi,
$$

where

$$
\mathbf{V}=c \int\left(C_{+}^{\uparrow *} \boldsymbol{\alpha} C_{+}^{\uparrow}+C_{-}^{\uparrow *} \boldsymbol{\alpha} C_{-}^{\uparrow}+C_{-}^{\downarrow *} \boldsymbol{\alpha} C_{-}^{\downarrow}\right) d^{3} \pi
$$

is the linear velocity of the average motion of electron, $l_{1}(\boldsymbol{\pi})=\left|C_{+}^{\uparrow *} \boldsymbol{\alpha} C_{-}^{\downarrow}\right|, \quad l_{2}(\boldsymbol{\pi})=\left|C_{+}^{\uparrow *} \boldsymbol{\alpha} C_{-}^{\uparrow}\right|$ and the phase term turns out to be

$$
\varphi(\boldsymbol{\pi})=\tan ^{-1} \frac{\operatorname{Im}\left(C_{+}^{\uparrow *} \boldsymbol{\alpha} C_{-}^{\downarrow}\right)}{\operatorname{Re}\left(C_{+}^{\uparrow *} \boldsymbol{\alpha} C_{-}^{\downarrow}\right)} .
$$

Integration of Eq. (23) in the weak field approximation reads

$$
<\boldsymbol{r}>\simeq \boldsymbol{r}_{\mathrm{i}}+\mathbf{V} t+\lambda_{c} \int\left\{l(\boldsymbol{\pi}) \sin \left[\left(\omega_{\mathrm{zbw}}+\omega_{c}\right) t+\varphi(\boldsymbol{\pi})\right]+l_{2}(\boldsymbol{\pi}) \sin \omega_{\mathrm{zbw}} t\right\} d^{3} \pi,
$$

\footnotetext{
${ }^{8}$ Note that, for a typical field of one Tesla, the right hand side of relation (20) is an order of about $10^{-63}$.
} 
where $l(\boldsymbol{\pi}) \simeq l_{1}(\boldsymbol{\pi})\left(1-\omega_{c} / \omega_{\text {zbw }}\right)$ and we assume $\boldsymbol{r}_{\mathrm{i}} \equiv \boldsymbol{r}_{\text {initial }}=0$. This relation, compared to the results with no external magnetic field, shows that the oscillatory part - which involves interference between the positive and negative energy states of electron - is now split into two terms. As it will be discussed bellow, within our approximation, the first term of the oscillatory part signifies the Zbw circular motion in the $x y$-plane with a shifted frequency, while the second term is responsible for Zbw in the $z$-direction with an intact frequency, as expected.

For the wave packet (21), the linear velocity term in $\langle\boldsymbol{r}\rangle$ vanishes. Whereas, up to the first order in $\pi / m c$, we have

$$
\begin{gathered}
l_{1 x}=l_{1 y} \simeq-f^{2}\left(\frac{\pi}{\pi_{o}}\right) \frac{\left(\pi_{x}^{2}+\pi_{y}^{2}\right)^{\frac{1}{2}}}{2 m c}, \quad l_{2 x}=l_{2 y}=l_{1 z} \simeq 0, \quad l_{2 z} \simeq-f^{2}\left(\frac{\pi}{\pi_{o}}\right) \frac{\pi_{z}}{2 m c}, \\
\varphi_{x}(\boldsymbol{\pi}) \simeq \tan ^{-1}\left(\frac{\pi_{y}}{\pi_{x}}\right), \quad \varphi_{y}(\boldsymbol{\pi}) \simeq \tan ^{-1}\left(-\frac{\pi_{x}}{\pi_{y}}\right) \quad \text { and } \quad \varphi_{z}(\boldsymbol{\pi})=0 .
\end{gathered}
$$

In the spherical coordinates, one gets

$$
\begin{aligned}
l_{1 x}=l_{1 y} \simeq-f^{2}\left(\frac{\pi}{\pi_{o}}\right) \frac{\pi \sin \theta}{2 m c}, \quad l_{2 x}=l_{2 y}=l_{1 z} \simeq 0, \quad l_{2 z} \simeq-f^{2}\left(\frac{\pi}{\pi_{o}}\right) \frac{\pi \cos \theta}{2 m c}, \\
\varphi_{x}(\boldsymbol{\pi}) \simeq \tan ^{-1}(\tan \varphi)=\varphi, \quad \varphi_{y}(\boldsymbol{\pi}) \simeq \tan ^{-1}(-\cot \varphi)=\varphi+\frac{\bar{\pi}}{2} \quad \text { and } \quad \varphi_{z}(\boldsymbol{\pi})=0 .
\end{aligned}
$$

Substituting these terms in relation (26), it becomes

$$
\begin{gathered}
<x>\simeq I^{\uparrow} \frac{\lambda_{c}}{2} \int_{0}^{2 \bar{\pi}} \sin \left[\left(\omega_{\mathrm{zbw}}+\omega_{c}\right) t+\varphi\right] d \varphi, \quad<y>\simeq I^{\uparrow} \frac{\lambda_{c}}{2} \int_{0}^{2 \bar{\pi}} \cos \left[\left(\omega_{\mathrm{zbw}}+\omega_{c}\right) t+\varphi\right] d \varphi \\
\text { and } \quad<z>\simeq J \lambda_{c} \sin \left(\omega_{\mathrm{zbw}} t\right),
\end{gathered}
$$

where

$$
I^{\uparrow} \equiv-2\left(1-\frac{\omega_{c}}{\omega_{\mathrm{zbw}}}\right) \int_{0}^{\infty} \int_{0}^{\bar{\pi}} \frac{f^{2}\left(\frac{\pi}{\pi_{o}}\right)}{2 m c} \pi^{3} \sin ^{2} \theta d \theta d \pi=-(8 \bar{\pi})^{-\frac{1}{2}} \frac{\lambda_{c}}{r_{o}}\left(1-\frac{\omega_{c}}{\omega_{\mathrm{zbw}}}\right)
$$

and

$$
J \equiv-\bar{\pi} \int_{0}^{\infty} \int_{0}^{\bar{\pi}} \frac{f^{2}\left(\frac{\pi}{\pi_{o}}\right)}{2 m c} \pi^{3} \sin 2 \theta d \theta d \pi=0 .
$$

As $\varphi$ is the azimuthal angle in the spherical momentum space, the nature of the integral in the $x y-$ plane over $\varphi$ restricts us to a fixed value, e.g. $\varphi_{o}$, in order to figure out the role of a specific Fourier decomposition in the Zbw circular motion. Of course, if one accomplishes the integrations over the full domain, then all oscillatory motions will disappear, and the center of the wave packet as a whole remains at rest, as expected. Hence, by considering those Fourier components in the $x y$-plane within $\varphi$ and $\varphi+d \varphi$, one gets

$$
<x>_{\varphi_{o}} \simeq \frac{\lambda_{c}}{2} \sin \left[\left(\omega_{\mathrm{zbw}}+\omega_{c}\right) t+\varphi_{o}\right] \quad \text { and } \quad<y>_{\varphi_{o}} \simeq-\frac{\lambda_{c}}{2} \cos \left[\left(\omega_{\mathrm{zbw}}+\omega_{c}\right) t+\varphi_{o}\right] .
$$

The notation $I^{\uparrow}$ - shown in Eq. (30) to be proportional to $\lambda_{c} / r_{o}$ - only determines the weight of Zbw relative to the degree of localization of electron. Eqs. (32) express a circular motion in the $x y$-plane, therefor one can conclude that the Zbw frequency of rotation for a spin-up electron in an external uniform static magnetic field changes by

$$
\omega_{\mathrm{zbw}}^{\uparrow} \rightarrow \omega_{\mathrm{zbw}}^{\uparrow}+\omega_{c}
$$

while the frequency for $\langle z\rangle$ remains unchanged, as expected. The same procedure for the spin-down state yields

$$
<x>\simeq I^{\downarrow} \frac{\lambda_{c}}{2} \int_{0}^{2 \bar{\pi}} \sin \left[\left(\omega_{\mathrm{zbw}}-\omega_{c}\right) t-\varphi\right] d \varphi, \quad<y>\simeq I^{\downarrow} \frac{\lambda_{c}}{2} \int_{0}^{2 \bar{\pi}} \cos \left[\left(\omega_{\mathrm{zbw}}-\omega_{c}\right) t-\varphi\right] d \varphi
$$


and $\quad<z>\simeq J \lambda_{c} \sin \left(\omega_{\mathrm{zbw}} t\right)$,

with

$$
I^{\downarrow} \equiv-2\left(1+\frac{\omega_{c}}{\omega_{\mathrm{zbw}}}\right) \int_{0}^{\infty} \int_{0}^{\bar{\pi}} \frac{f^{2}\left(\frac{\pi}{\pi_{o}}\right)}{2 m c} \pi^{3} \sin ^{2} \theta d \theta d \pi=-(8 \bar{\pi})^{-\frac{1}{2}} \frac{\lambda_{c}}{r_{o}}\left(1+\frac{\omega_{c}}{\omega_{\mathrm{zbw}}}\right) .
$$

Thus, for a spin-down electron, the frequency changes by

$$
\omega_{\mathrm{zbw}}^{\downarrow} \rightarrow \omega_{\mathrm{zbw}}^{\downarrow}-\omega_{c} .
$$

Obviously, the new Zbw frequencies reduce to the field free case in the absence of external magnetic field.

Motion of electron in an external uniform static magnetic field, employing the Heisenberg picture, has been discussed in Ref. [53]. They have also shown that the Zbw frequency, in the weak field approximation, changes to

$$
\omega_{\mathrm{zbw}} \pm \omega_{c}
$$

without explicitly distinguishing the spin-up from spin-down states. However, we have proved that one can assign a distinct modification to the $\mathrm{Zbw}$ frequency of each of these states given in relations (33) and (36). Though, one may still claim that $\omega_{\text {zbw }} \pm \omega_{c}$ term can intuitively be understood to stand for the spin-up/down states, respectively. But, such an interpretation has explicitly been revealed within our results. Besides, our outcome can be compared with the classical results via the BZ approach [25], that will be accomplished in the next section.

But before that, in order to gain further insight into the situation raised by the phenomenon of Zbw, one can examine the effect of an external uniform static magnetic field on some matrix elements that do not vanish. For this purpose, let us calculate the expectation value of the magnetic moment of a spin-up/down electron, that in the operator form it is [9]

$$
<\boldsymbol{\mu}>=\frac{e}{2 c}<\boldsymbol{r} \times \dot{\boldsymbol{r}}>=\frac{e}{2}<\boldsymbol{r} \times \boldsymbol{\alpha}>\rightarrow \frac{i e \hbar}{2}<\nabla_{\boldsymbol{\pi}} \times \boldsymbol{\alpha}>.
$$

Using the wave packet (21), one can easily show that, with the initial condition (17), the contribution from each Fourier component of expression (38) vanishes individually in the $x$ - and $y$-directions, but survives in the $z$-direction, as maybe expected. That is, from classical point of view, the $z$-component of (38), which is perpendicular to the circle of $\mathrm{Zbw}$ motion, is the only non-vanishing component of the magnetic moment. Straightforward calculations, using the employed approximations, reveal that one gets

$$
<\mu_{x}^{\uparrow}>=0, \quad<\mu_{y}^{\uparrow}>=0 \quad \text { and } \quad<\mu_{z}^{\uparrow}>=-\frac{|e| \lambda_{c}}{2}\left[1-\cos \left(\omega_{\mathrm{zbw}}+\omega_{c}\right) t\right]
$$

for the spin-up states, and

$$
<\mu_{x}^{\downarrow}>=0, \quad<\mu_{y}^{\downarrow}>=0 \quad \text { and } \quad<\mu_{z}^{\downarrow}>=\frac{|e| \lambda_{c}}{2}\left[1-\cos \left(\omega_{\mathrm{zbw}}-\omega_{c}\right) t\right]
$$

for the spin-down states. These results indicate that each Fourier wave contributes a circular motion about the direction of spin. Hence, one may again interpret that the intrinsic magnetic moment is a result of the (shifted) Zbw. Incidentally, the time dependant part of the magnetic moment, in relations (39) and (40), is a consequence of the fact that we have assumed that the spin of electron had initially been observed. This requirement leads to bring in the negative energy parts which do not vanish by letting the wave packet spread in space [9].

Furthermore, it would be instructive to employ the classical interpretation of spin $[9,15,18,19,20]$ via the intrinsic/microscopic angular momentum vector caused from the current produced by the local Zbw frequency when this frequency is shifted in the presence of an external magnetic field. For this 
purpose, by relations (29) and (34) when considering (30) and (35), one can claim that the radius of the Zbw circle is $r_{\mathrm{zbw}}=\lambda_{c}(1 \pm \varepsilon) / 2$, where $\varepsilon \equiv-\omega_{c} / \omega_{\mathrm{zbw}}$. And thus, the speed of electron around the center of mass will be

$$
v_{\mathrm{zbw}}=\left[\frac{\lambda_{c}}{2}(1 \pm \varepsilon)\right]\left[\omega_{\mathrm{zbw}}(1 \mp \varepsilon)\right]=c\left(1-\varepsilon^{2}\right) \simeq c .
$$

This result is consistent with the calculation of Zbw velocity via the Heisenberg picture, where the position operator $\boldsymbol{r}$ satisfies $\dot{\boldsymbol{r}}=i[H, \boldsymbol{r}] / \hbar=c \boldsymbol{\alpha}$. Hence, the intrinsic/microscopic angular momentum is

$$
s_{\mathrm{zbw}}=r_{\mathrm{zbw}}\left(m v_{\mathrm{zbw}}\right) \simeq \frac{\hbar}{2}(1 \pm \varepsilon) \simeq \frac{\hbar}{2(1 \mp \varepsilon)} .
$$

This indicates that the gyromagnetic $g$ factor of electron in the presence of an external magnetic field changes to $2(1 \mp \varepsilon)$, however the magnetic moment, relations (39) and (40), shows that the time average of the gyromagnetic $g$ factor in a period does not change.

On the other hand, if the spin of electron must remain unchanged, i.e. with the fixed value of $\hbar / 2$, then by assuming $v_{\mathrm{zbw}} \propto c$, (e.g. $v_{\mathrm{zbw}}=\zeta c$ ), the radius of the $\mathrm{Zbw}$ circle will be

$$
r_{\mathrm{zbw}}=\frac{v_{\mathrm{zbw}}}{\omega_{\mathrm{zbw}}(1 \mp \varepsilon)}=\frac{\lambda_{c}}{2}\left(\frac{\zeta}{1 \mp \varepsilon}\right) .
$$

Hence, the intrinsic/microscopic angular momentum is

$$
s_{\mathrm{zbw}}=\frac{\lambda_{c}}{2}\left(\frac{\zeta}{1 \mp \varepsilon}\right)\left(m v_{\mathrm{zbw}}\right)=\frac{\hbar}{2}\left(\frac{\zeta^{2}}{1 \mp \varepsilon}\right),
$$

which if it is supposed to remain $\hbar / 2$, one will get $\zeta \simeq 1 \mp \varepsilon / 2$. That is, in this view, $v_{\mathrm{zbw}} \simeq c(1 \mp \varepsilon / 2)$ and $r_{\mathrm{zbw}} \simeq \lambda_{c}(1 \pm \varepsilon / 2) / 2$.

\section{Frequency Shift via Classical Spinning Electron Approach}

As mentioned in the introduction, BZ devised [25, 33, 37] a new classical analogous model for the relativistic spinning electron in the ordinary space-time by introducing a classical Lagrangian as

$$
L=\frac{1}{2} i \alpha(\dot{\bar{z}} z-\bar{z} \dot{z})+p_{\mu}\left(\dot{x}^{\mu}-\bar{z} \gamma^{\mu} z\right)+e A_{\mu} \bar{z} \gamma^{\mu} z
$$

where $\mu=0,1,2,3, A^{\mu}$ is the electromagnetic potential, $\alpha$ is a constant with the dimension of action, $z$ and $\bar{z} \equiv z^{\dagger} \gamma^{0}$ are ordinary $\mathcal{C}^{4}$ bi-spinors which used as a second pair of conjugate classical spinorial variables, the speed of light, $c$, is set equal to one and dot indicates derivation with respect to the proper time, $\tau$, where $d / d \tau=\dot{x}^{\mu} \partial_{\mu}$.

The four Euler-Lagrange equations can be obtained with respect to variables $x^{\mu}, p^{\mu}, z$ and $\bar{z}$. By assuming $-\alpha=1=\hbar$ and adopting independent dynamical variables $x^{\mu}, \pi^{\mu}, v^{\mu}$ and $S^{\mu \nu}$, where $v^{\mu}$ is the 4 -velocity and $S^{\mu \nu} \equiv i \bar{z}\left[\gamma^{\mu}, \gamma^{\nu}\right] z / 4$ is the spin tensor met in the Dirac theory, the Euler-Lagrange equations are obtained within the classical approach to be [25]

$$
\dot{\pi}^{\mu}=e F^{\mu \nu} v_{\nu}, \quad \dot{x}^{\mu}=v^{\mu}, \quad \dot{v}^{\mu}=4 S^{\mu \nu} \pi_{\nu} \quad \text { and } \quad \dot{S}^{\mu \nu}=v^{\nu} \pi^{\mu}-v^{\mu} \pi^{\nu} .
$$

For a free electron (i.e. $A^{\mu}=0$ ), the equation of motion for 4 -velocity is derived to be [37]

$$
v^{\mu}=\frac{p^{\mu}}{m}-\frac{\ddot{v}^{\mu}}{4 m^{2}} .
$$

A general solution for this case has been shown to be $[11,33,36]$

$$
v^{\mu}=\frac{p^{\mu}}{m}+\left[v^{\mu}(0)-\frac{p^{\mu}}{m}\right] \cos \left(\omega_{\mathrm{zbw}} \tau\right)+\frac{\dot{v}^{\mu}(0)}{2 m} \sin \left(\omega_{\mathrm{zbw}} \tau\right) .
$$


The first term of this solution belongs to the rectilinear part of the velocity, the second and third ones indicate the Zbw motion.

For a spinning electron in an external electromagnetic field, third equation of Eqs. (46) indicates that the general solution represents a helical motion in the ordinary 3-space. In fact, the equation of motion from Eqs. (46) is derived to be [25]

$$
\ddot{v}^{\mu}-4 m \pi^{\mu}+4 m^{2} v^{\mu}+4 e v^{\mu} F_{\alpha \beta} S^{\alpha \beta}-4 e S^{\mu \nu} F_{\nu \beta} v^{\beta}=0,
$$

where $F^{\mu \nu}$ is the electromagnetic tensor. Writing the $x$ - and $y$-components of Eq. (49) for an electron with the $z$-component of spin in an external uniform static magnetic field along the $z$-direction (in which the only nonzero components of the electromagnetic tensor are $F^{21}=-F^{12}=B$ ), then deriving with respect to $\tau$ while using the first equation of (46) results in [25]

$$
\begin{aligned}
& \dddot{v}_{x}+4\left(m^{2}-3 e s_{z} B\right) \dot{v}_{x}-4 m e B v_{y}=0, \\
& \dddot{v}_{y}+4\left(m^{2}-3 e s_{z} B\right) \dot{v}_{y}+4 m e B v_{x}=0 .
\end{aligned}
$$

These equations describe a uniform circular motion in the $x y$-plane whose angular velocities must satisfy the algebraic equation [25]

$$
\omega^{3}-4\left(m^{2}-3 e s_{z} B\right) \omega+4 m e B=0,
$$

or equivalently, with our notations,

$$
\omega^{3}-\omega_{\mathrm{zbw}}^{2} \omega \pm 3 \varepsilon \omega_{\mathrm{zbw}}^{2} \omega+\varepsilon \omega_{\mathrm{zbw}}^{3}=0,
$$

where the upper (lower) sign represents the spin-up (down) state. Solutions of (52), by the weak field approximation and up to the first-order terms in $\varepsilon$, have been asserted to be $[25]^{9}$

$$
\omega_{1} \simeq \omega_{c}(1 \pm 3 \varepsilon), \quad \omega_{2} \simeq \omega_{\mathrm{zbw}}\left(1 \mp \frac{3}{2} \varepsilon\right) \quad \text { and } \quad \omega_{3} \simeq-\omega_{\mathrm{zbw}}\left(1 \mp \frac{3}{2} \varepsilon\right) .
$$

Frequency $\omega_{1}$ has been regarded [25] as the usual rotational frequency of electron in an external magnetic field, which due to the Zbw, differs from that of spinless particles. Namely, it shows the effect of spin on the cyclotron frequency. It has also been claimed [25] that the small predicted deviation to the cyclotron frequency can be detected by their suggested device. This argument nearly explains the purpose of Ref. [25]. Meanwhile, the other two solutions, $\omega_{2}$ and $\omega_{3}$, have been described as modified forms of $\omega_{\mathrm{zbw}}$ which, due to the smallness of $\varepsilon$, have essentially been considered [25] identical to $\omega_{\text {zbw }}$ without further investigation.

In this work, we purpose to probe these terms more closely. Actually, we are interested in investigating effects of external magnetic fields on the Zbw frequency itself. Of course, there is no doubt that due to the smallness of variations involved in this issue, one has little hope of detection by the current experiments, unless innovative device will emerge. ${ }^{10}$ Examples fall short of the level of this effect, even detection of the $\omega_{\mathrm{zbw}}$ itself for free electron are outside of the current experimental and technological reach $[13,50,52]$. Unless, e.g., they are indirectly in the response of electron to external fields and/or a kind of condition (e.g. a resonance condition) employed to minimize the Zbw frequency. For example, the Darwin term in the Hamiltonian of an atomic electron comes from the Zbw motion which makes electron sensitive to the potential of the nuclei in its average position [2]-[8]. Furthermore, to grasp an intuitive sense, one may recall that the Zbw frequency of oscillation is too

\footnotetext{
${ }^{9}$ Again, we have rewritten their solutions with our notations.

${ }^{10}$ Interested readers in related experimental techniques may consult Ref. [57] (and references therein), where they have measured the electron's $g$ factor. Also, see Refs. [13, 20], [44]-[52] for more recent theoretical studies for experimentally observing and/or experimental devices on the phenomenon of Zbw. For example, an optical lattice scheme, which may permit experimental observation of the Zbw with ultra-cold neutral atoms, has been proposed in Ref. [50], in where they also claim that the phenomenon of Zbw will occur at experimentally accessible frequencies and amplitudes.
} 
high and hence the period of the time involved is too low, whereas the time measurement sensitivity limits are generally known to be about $10^{-16}$ s [58].

Nevertheless, following our purpose while probing the solutions $\omega_{2}$ and $\omega_{3}$, we have noticed that these two solutions of (53), are very rough approximations. Actually, the more accurate solutions $\omega_{2}$ and $\omega_{3}$, after solving equation (52), are

$$
\omega_{2} \simeq \omega_{\mathrm{zbw}}\left(\begin{array}{c}
1-2 \varepsilon \\
1+\varepsilon
\end{array}\right) \quad \text { and } \quad \omega_{3} \simeq-\omega_{\mathrm{zbw}}\left(\begin{array}{c}
1-\varepsilon \\
1+2 \varepsilon
\end{array}\right),
$$

where again the upper (lower) value represents solution for the spin-up (down) state. These solutions contradict with the solutions derived from the quantum approaches, i.e. the approach employed in this work via the expectation value and the one used in Ref. [53] via the Heisenberg picture.

The more accurate solutions (54) lead to some discrepancies. In fact, regarding solution (48) and via the BZ spinning particle model, Salesi and Recami have associated $\left(-\omega_{\mathrm{zbw}}\right)$ with antiparticles and $\omega_{\mathrm{zbw}}$ with particles [59]. This way, they have concluded that the only change which happens to the Zbw part of the motion via $\omega_{\mathrm{zbw}} \rightarrow-\omega_{\mathrm{zbw}}$ would be conversion of counterclockwise motion to the clockwise one. It means that solution (48) should treat electrons and positrons, other than assigning a different direction of rotation to their Zbw motions, in the same spin states. In this merit, it has also been inferred [25] that $\omega_{2}$ and $\omega_{3}$ are generalized features of $\omega_{\mathrm{zbw}}$ and $\left(-\omega_{\mathrm{zbw}}\right)$, respectively.

Now, let us investigate this issue for the solutions (54), in which they can be rewritten for electron and positron with the spin-up as ${ }^{11}$

$$
\begin{aligned}
\omega_{\mathrm{zbw}}^{\uparrow} & \rightarrow \omega_{2}^{\uparrow} \simeq \omega_{\mathrm{zbw}}^{\uparrow}-2 \varepsilon \omega_{\mathrm{zbw}}^{\uparrow} \\
\left(-\omega_{\mathrm{zbw}}^{\uparrow}\right) & \rightarrow \omega_{3}^{\uparrow} \simeq\left(-\omega_{\mathrm{zbw}}^{\uparrow}\right)-\varepsilon\left(-\omega_{\mathrm{zbw}}^{\uparrow}\right),
\end{aligned}
$$

while with the spin-down as

$$
\begin{aligned}
\omega_{\mathrm{zbw}}^{\downarrow} & \rightarrow \omega_{2}^{\downarrow} \simeq \omega_{\mathrm{zbw}}^{\downarrow}+\varepsilon \omega_{\mathrm{zbw}}^{\downarrow} \\
\left(-\omega_{\mathrm{zbw}}^{\downarrow}\right) & \rightarrow \omega_{3}^{\downarrow} \simeq\left(-\omega_{\mathrm{zbw}}^{\downarrow}\right)+2 \varepsilon\left(-\omega_{\mathrm{zbw}}^{\downarrow}\right) .
\end{aligned}
$$

These relations show that the existence of an external magnetic field breaks the usual symmetries which hold for free particles and antiparticles. That is, the spin-up electron experiences a new Zbw frequency while the one for the spin-up positron is different, and vice versa for the spin-down states. In addition, the Zbw frequency of an electron (positron) exposed to an external magnetic field behaves oddly, and the absolute value of frequency shift depends on the orientation of spin. Indeed, the Zbw frequency of the spin-up particle changes twice that of the spin-down, and vice versa for antiparticles. Therefore, the usual symmetry of free particles and antiparticles states, which is valid in the electromagnetic interactions, is broken. More obvious, it means that the well-known CP symmetry, which is respected in the quantum electrodynamics, ${ }^{12}$ is violated, however, the quantum mechanical symmetries might not necessarily hold in the classical level.

Incidentally, if one repeats the calculations of Sec. 2 with the same initial state but for positron instead of electron, namely

$$
\Psi(\mathbf{r}, 0)=\left(\begin{array}{l}
0 \\
0 \\
1 \\
0
\end{array}\right) f\left(\frac{r}{r_{o}}\right)
$$

instead of relation (17), one will get

$$
\omega_{\mathrm{zbw}}^{\uparrow} \rightarrow \omega_{\mathrm{zbw}}^{\uparrow}-\omega_{c}
$$

\footnotetext{
${ }^{11}$ Note that, according to our convention $\varepsilon$ has a negative value.

${ }^{12}$ See standard textbooks on the quantum field theory, e.g. Ref. [6].
} 
and

$$
\omega_{\mathrm{zbw}}^{\downarrow} \rightarrow \omega_{\mathrm{zbw}}^{\downarrow}+\omega_{c}
$$

instead of shifts (33) and (36). Now, if one can justify - e.g. based on the initial states (17) and (57) - the application of the classical interpretation of Ref. [59] for the expectation value approach, then one will easily be able to show that this approach holds the above discussed symmetries. ${ }^{13}$ In another words, our results, relations (33), (36), (58) and (59), indicate that the amount of frequency shifts are opposite in the spin-up/down states and in electron/positron cases, which are consistent with the CP symmetry.

\section{Discussion and Conclusion}

Much effort in the literature has been devoted to putting forward a consistent theory to describe the spin of electron. Among them, Huang [9] used the Dirac equation to tackle this issue and BZ [33] introduced a new classical model, which is believed to be the most satisfactory picture of a classical spinning electron constituting a natural classical limit of the Dirac equation [25, 36].

In this work, we have employed the expectation value quantum approach [9] to investigate the effects of an external magnetic field on the Zbw frequency of electron. We have found that, in the non-relativistic weak field approximation, the Zbw frequency in the presence of an external uniform static magnetic field changes according to relations (33) and (36) for electrons, and relations (58) and (59) for positrons. Actually, the individual Fourier components of the position expectation value of an electron with the certain initial conditions do not vanish, and indeed lead to an interpretation in terms of a shifted Zbw frequency. Furthermore, not only each of the Fourier wave contributes a circular motion in the plane perpendicular to the direction of electron spin with radius $\lambda_{c} / 2$, rather the shifted Zbw frequency depends equally on the orientation of the direction as well, i.e. the spin-up or down. Our results are exactly the same as those derived in Ref. [53] via the Heisenberg picture. However, contrary to Ref. [53], we have explicitly shown the distinct modifications for the spin-up and spin-down states, as one may intuitively expect. Besides, in order to compare the classic and quantum approaches to the Zbw in an external magnetic field, this characteristic of our results are more appropriate.

The BZ classical approach has been generalized [25] to include the case of an electron in the presence of an external uniform static magnetic field. Hence, it is shown that in the weak field approximation, the motion of electron is described by the three characteristic frequencies, where one of them reflects the effect of spin on the cyclotron frequency and the other two are the modified forms of $\omega_{\mathrm{zbw}}$. We have noticed that the latter two derived frequencies are very rough approximations. Thus, we have indicated the more accurate solutions, solutions (54). However, contrary to the quantum approaches - the approach employed in this work via the expectation value and the one used in Ref. [53] via the Heisenberg picture - the solutions (54) are controversial and break the usual symmetry of free particles and antiparticles states. We have made this point more obvious by writing the solutions (54) via the BZ [59] spinning particle and antiparticle interpretation. Indeed, these solutions, relations (55) and (56), indicate that the frequency shifts depend nontrivially on the orientation of spin and also on whether one is dealing with particles or antiparticles. Hence, CP violation occurs in the classical electrodynamics domain. Therefore, regarding the Zbw frequency of electron, one may conclude that the BZ classical approach in the presence of an external magnetic field is unlikely to correctly describe the spin of electron, while the quantum approach does. That is, the quantum approach respects the CP symmetry, as expected.

\footnotetext{
${ }^{13}$ However, one cannot make such a classical energy separation within the relativistic quantum approaches, see, e.g., the wave packet $(21)$.
} 


\section{Appendix}

Using the initial state (17) and approximations (19), (20) and $\left(\pi_{x} h_{ \pm}^{\uparrow \downarrow} \simeq h_{ \pm}^{\uparrow \downarrow} \pi_{x}\right)$, the exact coefficients in relation (15) are found roughly to be

$$
\begin{aligned}
\mathcal{A} & =\frac{h_{-}^{\uparrow} h_{+}^{\downarrow} h_{-}^{\downarrow}+h_{-}^{\uparrow} h_{+}^{\downarrow} h_{-}^{\downarrow} K_{1}^{2} \pi_{z}^{2}+h_{-}^{\downarrow} K_{1} K_{2} \pi_{-} h_{+}^{\downarrow} \pi_{+} h_{-}^{\uparrow}}{\Gamma} f\left(\pi / \pi_{o}\right) \simeq \frac{f\left(\pi / \pi_{o}\right)}{h_{+}^{\uparrow}}, \\
\mathcal{B} & =\frac{-h_{-}^{\downarrow} K_{2}^{2} \pi_{+} h_{-}^{\uparrow} \pi_{z} h_{+}^{\uparrow}+h_{-}^{\uparrow} h_{-}^{\downarrow} K_{1} K_{2} \pi_{z} \pi_{+} h_{+}^{\uparrow}}{\Gamma} f\left(\pi / \pi_{o}\right) \simeq 0, \\
\mathcal{C} & =-\frac{K_{1}^{2} K_{2} \pi_{-} h_{+}^{\downarrow} \pi_{z} h_{-}^{\downarrow} \pi_{+} h_{+}^{\uparrow}+h_{+}^{\uparrow} h_{+}^{\downarrow} h_{-}^{\downarrow} K_{1}^{2} K_{2} \pi_{z}^{3}+h_{+}^{\uparrow} h_{+}^{\downarrow} h_{-}^{\downarrow} K_{2} \pi_{z}}{\Gamma} f\left(\pi / \pi_{o}\right) \\
& \simeq \frac{-K \pi_{z}}{h_{-}^{\uparrow}} f\left(\pi / \pi_{o}\right), \\
\mathcal{D} & =-\frac{h_{+}^{\uparrow} h_{+}^{\downarrow} K_{1} K_{2}^{2} \pi_{z}^{2} \pi_{+} h_{-}^{\uparrow}+K_{1} K_{2}^{2} \pi_{+} h_{+}^{\uparrow} \pi_{-} h_{+}^{\downarrow} \pi_{+} h_{-}^{\uparrow}+h_{-}^{\uparrow} h_{+}^{\downarrow} K_{2} \pi_{+} h_{+}^{\uparrow}}{\Gamma} f\left(\pi / \pi_{o}\right) \\
& \simeq \frac{-K \pi_{+}}{h_{-}^{\downarrow}} f\left(\pi / \pi_{o}\right),
\end{aligned}
$$

where

$$
\begin{aligned}
\Gamma \equiv & h_{+}^{\uparrow} h_{-}^{\uparrow} h_{+}^{\downarrow} h_{-}^{\downarrow}+h_{+}^{\uparrow} h_{+}^{\downarrow} K_{1}^{2} K_{2}^{2} \pi_{z}^{2} \pi_{-} h_{-}^{\downarrow} \pi_{+} h_{-}^{\uparrow}+h_{-}^{\uparrow} h_{-}^{\downarrow} K_{1}^{2} K_{2}^{2} \pi_{z}^{2} \pi_{-} h_{+}^{\downarrow} \pi_{+} h_{+}^{\uparrow} \\
& +h_{+}^{\uparrow} h_{-}^{\uparrow} h_{+}^{\downarrow} h_{-}^{\downarrow} K_{1}^{2} \pi_{z}^{2}+h_{+}^{\uparrow} h_{-}^{\uparrow} h_{+}^{\downarrow} h_{-}^{\downarrow} K_{1}^{2} K_{2}^{2} \pi_{z}^{4}+h_{+}^{\uparrow} h_{-}^{\uparrow} h_{+}^{\downarrow} h_{-}^{\downarrow} K_{2}^{2} \pi_{z}^{2} \\
& +h_{+}^{\uparrow} h_{-}^{\downarrow} K_{1} K_{2} \pi_{-} h_{+}^{\downarrow} \pi_{+} h_{-}^{\uparrow}+K_{1}^{2} K_{2}^{2} \pi_{-} h_{+}^{\downarrow} \pi_{-} h_{-}^{\downarrow} \pi_{+} h_{-}^{\uparrow} \pi_{+} h_{+}^{\uparrow}+h_{-}^{\uparrow} h_{+}^{\downarrow} K_{1} K_{2} \pi_{+} h_{+}^{\uparrow} \pi_{-} h_{-}^{\downarrow} .
\end{aligned}
$$

\section{Acknowledgement}

We thank the Research Office of the Shahid Beheshti University for financial support. M.Z.-A. would like to appreciate his supervisor M.F. for very useful comments which have made the completion of this work possible.

\section{References}

[1] P.A.M. Dirac, Proc. Roy. Soc. A117 (1928), 610-624; ibid A118 (1928), 351-361.

[2] P.A.M. Dirac, The Principles of Quantum Mechanics, (Oxford University Press, Oxford, 4th ed. 1958).

[3] M.E. Rose, Relativistic Electron Theory, (John Wiley \& Sons, New York, 1961).

[4] J.D. Bjorken \& S.D. Drell, Relativistic Quantum Mechanics, (McGraw-Hill, New York, 1964).

[5] J.J. Sakurai, Advanced Quantum Mechanics, (Pearson Education, Inc., Delhi India, 1967).

[6] C. Itzykson \& J.-B. Zuber, Quantum Field Theory, (McGraw-Hill, Singapore, 1980).

[7] W. Greiner, Relativistic Quantum Mechanics, (Springer-Verlag, Berlin, 1990).

[8] E. Merzbacher, Quantum Mechanics, (John Wiley \& Sons, New York, 3rd ed. 1998).

[9] K. Huang, Am. J. Phys. 20 (1952), 479-484.

[10] E. Schrödinger, Sitz. Preuss. Akad. Wiss. Phys. Math. Kl. 24 (1930), 418-428; ibid 3 (1931), 1.

[11] A.O. Barut \& A.J. Bracken, Phys. Rev. D23 (1981), 2454-2463. 
[12] T.D. Newton \& P. Wigner, Rev. Mod. Phys. 21 (1949), 400-406.

[13] T.M. Rusin \& W. Zawadzki, J. Phys.: Cond. Matter 19 (2007), 136219 [18 pages]; Phys. Rev. B76 (2007), 195439 [7 pages].

[14] D. Bender \& et al., Phys. Rev. D30 (1984), 515-527.

[15] D. Hestenes, Found. Phys. 23 (1993), 365-387.

[16] W. Gordon, Z. Physik 50 (1927), 630-632.

[17] J. Frenkel, Wave Mechanics, Advanced General Theory, (Oxford University Press, London, 1934).

[18] A.O. Barut \& A.J. Bracken, Phys. Rev. D24 (1981), 3333-3334.

[19] D. Hestenes, Found. Phys. 20 (1990), 1213-1232.

[20] D. Hestenes, Found. Phys. 40 (2010), 1-54.

[21] M. Mathissen, Acta Phys. Polon 6 (1937), 163-200; ibid (1937), 218.

[22] H. Hönl, Ann. Physik 33 (1938), 565-585.

[23] J.W. Weyssenhof, Nature 141 (1938), 328-329; Acta Phys. Polon 9 (1947), 47-53.

[24] J. Brandmüller, Naturwiss. 38 (1951), 139-139.

[25] G. Salesi \& E. Recami, Phys. Lett. A267 (2000), 219-224.

[26] M.H.L. Pryce, Proc. Roy. Soc. London A195 (1948), 62-81.

[27] L.L. Foldy \& S.A. Wouthuysen, Phys. Rev. 78 (1950), 29-36.

[28] S. Tani, Progr. Theor. Phys. 6 (1951), 267-285.

[29] H. Feshbach \& F. Villars, Rev. Mod. Phys. 30 (1958), 24-25.

[30] A.O. Barut \& S. Malin, Rev. Mod. Phys. 40 (1968), 632-651.

[31] J.A. Lock, Am. J. Phys. 47 (1979), 797-802.

[32] P. Krekora, Q. Su \& R. Grobe, Phys. Rev. Lett. 93 (2004), 043004 [4 pages].

[33] A.O. Barut \& N. Zanghi, Phys. Rev. Lett. 52 (1984), 2009-2012.

[34] E. Recami \& G. Salesi, Phys. Rev. A57 (1998), 98-105.

[35] G. Salesi, Int. J. Mod. Phys. A17 (2002), 347-374.

[36] A.O. Barut \& M. Pavšič, Class. Quant. Grav. 4 (1987), L131-L136.

[37] E. Recami \& G. Salesi, "Field theory of the electron: spin and Zitterbewegung", in Particles, Gravity and Space-Time, Ed. P.I. Pronin \& G.A. Sardanashvily (World Scientific, Singapore, 1996), pp. 345-368, [hep-th/9508168].

[38] M. Pavšič, E. Recami \& W.A. Rodrigues Jr., Hadronic J. 18 (1995), 97-118.

[39] W. Zawadzki, in Optical Properties of Solides, Ed. E.D. Haidemenakis (Gordon \& Breach, New York, 1970), p. 179.

[40] F. Cannata, L. Ferrari \& G. Russo, Solid State Commun. 74 (1990), 309-312. 
[41] L. Ferrari \& G. Russo, Phys. Rev. B42 (1990), 7454-7461.

[42] W. Zawadzki, in High Magnetic Fields in the Physics of Semiconductors II, Ed. G. Landwehr \& W. Ossau (World Scientific, Singapore, 1997), p. 755.

[43] G. Salesi, Int. J. Mod. Phys. A20 (2005), 2027-2036.

[44] Z.F. Jiang, R.D. Li, S.C. Zhang \& W.M. Liu, Phys. Rev. B72 (2005), 045201 [5 pages].

[45] W. Zawadzki, Phys. Rev. B72 (2005), 085217 [4 pages].

[46] W. Zawadzki, "One-dimensional semirelativity for electrons in carbon nanotubes", condmat/0510184 [4 pages].

[47] J. Schliemann, D. Loss \& R.M. Westervelt, Phys. Rev. Lett. 94 (2005), 206801 [4 pages]; Phys. Rev. B73 (2006), 085323 [9 pages].

[48] M.I. Katsnelson, Eur. Phys. J. B51 (2006), 157-160.

[49] J. Cserti \& G. Dávid, Phys. Rev. B74 (2006), 172305 [4 pages].

[50] J.Y. Vaishnav \& C.W. Clark, Phys. Rev. Lett. 100 (2008), 153002 [4 pages].

[51] P. Brusheim \& H.Q. Xu, "Catching the Zitterbewegung", con-mat/0810.2186 [4 pages].

[52] L. Lamata, J. León, T. Schätz \& E. Solano, Phys. Rev. Lett. 98 (2007), 253005 [4 pages].

[53] M. Villavicencio \& J.A.E. Roa-Neri, Eur. J. Phys. 21 (2000), 119-123.

[54] J.W. van Holten, Physica A. 182 (1992), 279-292.

[55] J.W. van Holten, "Relativistic dynamics of spin in strong external fields", hep-th/9303124 [11 pages].

[56] H. Thirring, Principles of Quantum Electrodynamics, (Academic Press, New York, 1958).

[57] R.S. Van Dyck Jr., P.B. Schwinberg \& H.G. Dehmelt, Phys. Rev. D34 (1986), 722-736.

[58] T.P. Heavner, S.R. Jefferts, E.A. Donley, J.H. Shirley \& T.E. Parker, Metrologia 42 (2005), 411-422.

[59] G. Salesi \& E. Recami, Found. Phys. Lett. 10 (1997), 533-546. 